\title{
Structure of Tetrahymena CCT $\theta$ gene and its expression under colchicine treatment $^{1}$
}

\author{
Célia Domingues ${ }^{a}$, Helena Soares ${ }^{\mathrm{a}, \mathrm{b}}$, Claudina Rodrigues-Pousada ${ }^{\mathrm{a}}$, Luisa Cyrne ${ }^{\mathrm{a}, \mathrm{c}, *}$ \\ a Instituto Gulbenkian de Ciência, Apartado 14, P-2781 Oeiras, Portugal \\ b Escola Superior de Tecnologia de Saúde de Lisboa, 1700 Lisbon, Portugal \\ c Departamento de Química e Bioquímica, Faculdade de Ciências, Universidade de Lisboa, 1749-016 Lisbon, Portugal
}

Received 12 April 1999; received in revised form 18 June 1999; accepted 28 June 1999

\begin{abstract}
We report here the cloning and the characterization of the Tetrahymena pyriformis chaperonin-containing-TCP1 theta gene (ТpCCT $\theta$ ), an orthologue of the mouse chaperonin gene CСТ $\theta$. ТрССТ $\theta$ gene is interrupted by eight introns, ranging in size between 91 and 419 nucleotides, and encodes a protein consisting of 540 amino acid residues $(59.1 \mathrm{kDa})$, with a putative $\mathrm{p} I$ of 5.73. The amino acid sequence of TpCCT $\theta$ reveals $39.4-46.0 \%$ identity with the sequences of Candida albicans and mouse CCT $\theta$ subunits and $28.0-32.6 \%$ identity with the other TpCCT subunits known so far. We have studied the expression of this gene in exponentially growing Tetrahymena cells and in cells treated with colchicine for different times. The steadystate levels of CCT $\theta$ mRNA rapidly decrease in the first $30 \mathrm{~min}$ of colchicine treatment. Interestingly, treatment for subsequent $60 \mathrm{~min}$ gives expression levels higher than those found in exponentially growing cells. (C) 1999 Elsevier Science B.V. All rights reserved.
\end{abstract}

Keywords: CCT $\theta$-chaperonin gene; Ciliated protozoan; Gene expression; Colchicine

Inside cells the majority of newly synthesized proteins require a class of proteins, generally designated as molecular chaperones, to achieve their correct three-dimensional structure [1]. One of the most studied classes of the molecular chaperones is the chaperonins, which recognize and bind unfolded polypeptides to prevent premature folding and aggregation [2]. Based on sequence homology chaperonins can be grouped in two subfamilies. The first, Hsp60 family, is represented by GroEL in the eubacterial cytoplasm, Rubisco binding protein in chloroplast

\footnotetext{
* Corresponding author. Fax: +351-1-440-7970; E-mail: mcyrne@igc.gulbenkian.pt

1 Accession number: AF136446.
}

and its mitochondrial homologue Hsp60 [3]. The second family is represented by TF55, thermosome and the CCT complex, being found in archaebacteria and eukaryotic cytoplasm, respectively [4-6]. The eukaryotic cytosolic chaperonin, named CCT (Chaperonin Containing TCP-1 ( $t$-complex polypeptide 1)) is a large hetero-oligomeric complex of about $900 \mathrm{kDa}$, composed of eight different but related polypeptides, namely CCT $\alpha$, CCT $\beta \ldots C C T \xi$, of apparent molecular mass ranging from 52 to $65 \mathrm{kDa}$, arranged in two stacked rings. In mouse and yeast the genes encoding the eight CCT-subunit polypeptides have been isolated, whereas several CCT-subunit orthologues were already identified in a variety of organisms ranging from ciliates to human cells [7-9]. Together with co-factors and possibly other components still 


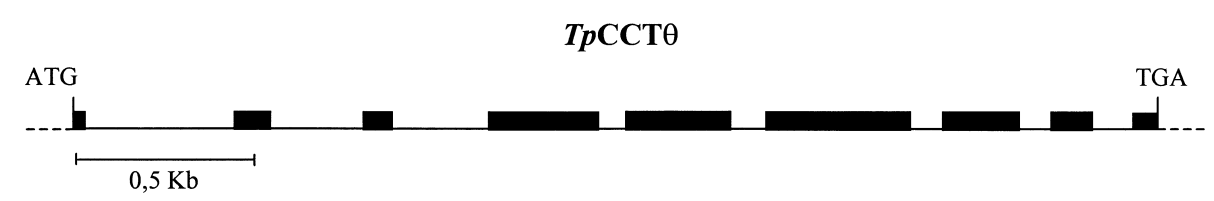

Fig. 1. Gene structure of the Tetrahymena CCT $\theta$ subunit. Schematic representation of the structure of the TpCCT $\theta$ gene. The boxes indicate exons and introns are shown by lines. ATG and TGA represent the first codon of the coding region and the stop codon, respectively.

remaining to be described, CCT mediates the folding or refolding of tubulins [10,11] and actins [12], and perhaps other unknown proteins. Studies of expression of some of the CCT-subunit genes show that the $\mathrm{CCT} \alpha$ is highly expressed in testis [13], embryos of early stages and rapidly growing cells in tissue culture $[9,14]$, suggesting that CCT is involved in cellcycle regulated events. Several studies indicate a relationship between the chaperonin and the cytoskeleton. Analysis of temperature-sensitive mutants of yeast CCT subunits shows abnormal microtubular structures [15] and disruption of actin microfilaments [16]. In yeast, all the CCT-subunit genes are essential and none of them when overexpressed rescues the mutation in any of the other, suggesting that each of the CCT subunits has distinct function. The assumption that tubulin is one of the major substrates of CCT raises the hypothesis that this chaperonin could be a new factor involved in generating MT function diversity. Indeed, microtubules are dynamic polymers that participate in a wide variety of cellular functions, such as cytoskeleton organization, intracellular transport and cell division, as well as flagellar and ciliary movement [17]. MTs are composed of $\alpha$ and $\beta$-tubulin heterodimers that constitute their basic structural unit. Tubulins have binding sites for a variety of antimitotic agents as, e.g., colchicine. This agent is known to affect the dynamics of microtubule polymerization both in vitro and in vivo $[18,19]$. It has been described that colchicine binds to the tubulin heterodimer changing its conformation and in consequence reduces the ability of the tubulin to dissociate from the microtubule end, hence impairing the capacity of the microtubule end to accept additional tubulin heterodimers [18].

In the ciliate Tetrahymena three members of the CCT-subunit gene family, namely TpCCT $\alpha$ [20],

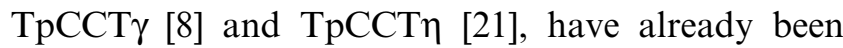
cloned and characterized. These genes exhibit a co- ordinate response and are up-regulated with the tubulin genes during cilia regeneration and during active cell division, indicating a possible role of these subunits in these processes.

In the present work another member of the Tetrahymena CCT gene family, ТрCCT $\theta$, was characterized, and its expression was studied in reciliating cells and cells treated with colchicine at different times.

Degenerate primers were designed taking into account the sequence corresponding to the amino acid sequence conserved among all CCT subunits NGATI (CCTnd) and the specific sequence DDIERAV/IV (CCT8c) present in all CCT $\theta$ subunits so far known (CCTnd: 5'-GCCTCTAGAAAYGAYGGTGCYACYATY-3' and CCT8c: 5'-CRAYRGCTCTTTCRATRTCRTC- $3^{\prime}$ ). These primers were used to amplify Tetrahymena pyriformis genomic DNA usingpolymerase chain reaction (PCR) cycling described previously [21]. The PCR products with 0.75 and $1.5 \mathrm{~kb}$ were cloned into pUC19 and sequenced. Given that the $1.5 \mathrm{~kb}$ product encodes the predicted amino acid sequence sharing a significant similarity with its mouse counterpart [22], the recombinant plasmid was named pCCT 05 . The fragment cloned in pCCT $\theta 5$ was labelled using the Megaprimer DNA labelling system and $\left[\alpha_{-}{ }^{32} \mathrm{P}\right] \mathrm{dATP}$ $(3000 \mathrm{Ci} / \mathrm{mmol})$ (Amersham) following the manufacturer's instructions, and then used to screen a $T$. pyriformis Sau 3AI genomic library and followed as essentially described in [8]. The DNA of one of the positive phage, named Tp $\lambda 6 \mathrm{CCT} \theta$, was analysed by restriction mapping following by subcloning the restriction fragments into pUC19. In order to sequence these fragments, sequential deletions were generated using a double-stranded Nested Detection kit (Pharmacia). Sequencing was performed on denatured plasmid DNA using a T7 sequencing kit (Pharmacia) according to the supplier's instructions. Sequence analysis was carried out using the GCG package 
(1991) (Genetics Computer Group) and the program DNASIS 5.0 (Hitachi-LKB).

Sequence analysis of the TpCCT $\theta$ subunit gene has shown that the coding region of this gene is interrupted by eight introns, as shown in Fig. 1. The introns are always flanked by the invariant dinucleotides GT and AG. TpCCT $\theta$ introns range in size between 91 and 419 nucleotides, which is similar to that found for the other TpCCT genes so far characterized. However, this gene contains one or two additional introns when compared to those of TpCCT $\eta$, ТрСCT $\alpha$ and TpCCT $\gamma$ genes, respectively [20]. The ТрCCT $\theta$ gene encodes a protein consisting of 540 amino acid residues, with a predicted molecular mass of about $59.1 \mathrm{kDa}$ and a putative $\mathrm{p} I$ of 5.73. The predicted TpCCT $\theta$ polypeptide has an identical size to CCT $\theta$ subunits from distinct organisms so far studied ( $T p$ 540, Sc 568, Sp 546, Ca 546, Mm 548 and $H h$ 548) with the exception for Saccharomyces cerevisiae CCT $\theta$ subunit, which has 28 extra amino acid residues. The amino acid sequence comparison among CCT $\theta$ subunits from different species reveals a high degree of sequence similarity: $68.7 \%$ with S. cerevisiae (P47079), 68.8\% with Schizosaccharomyces pombe (P78921), 63.8\% with Candida albicans (P47828) and 69.9\% with mouse and humans [22]; and a lower degree of sequence identity, with $41.9 \%, 42.3 \%, 39.4 \%$ and $46.0 \%$, respectively. When this type of analysis is limited to Tetrahymena CCT subunits the degree of sequence identity and similarity decreases. Indeed a similarity of $56.9 \%, 55.3 \%$ and $53.2 \%$ was obtained when compared with the $\mathrm{CCT} \alpha, \mathrm{CCT} \gamma$ and CCT $\eta$ subunits, whereas the identity values were $32.6 \%, 28.0 \%$ and $29.1 \%$, respectively. These results support the idea that although the different CCT subunits are structurally related, they are more conserved interspecies than between intraspecies. Fig. 2 shows the alignment of the predicted amino acid sequence of Tetrahymena ТрСCT $\theta$ and those of known CCT $\theta$ subunits from other organisms. This alignment reveals three conserved regions between all CCT subunits, as well as with other members of the chaperonin family [14]: the motifs TNDGATIL (TSDAATIM) (positions 64-71), GDGTTSV (GDATNLV) (positions 95-102) and V(P/A)GGG (AAGAG) (positions 408-412). Interestingly, a highly conserved region DDIERAIDDGVN (positions 385-396) that is not shared by the other CCT subunits and seems to be exclusively present in CCT $\theta$ subunits. It is possible that this sequence may be associated to a specific function of the CCT $\theta$ subunits inside the hetero-oligomeric complex of the chaperonins.

Previously we were able to show that $T$. pyriformis contains the hetero-oligomeric complex CCT and to assign in the two-dimensional (2-D) gel, using a set of polyclonal antibodies, the protein spots to the $\mathrm{CCT} \alpha, \mathrm{CCT} \beta, \mathrm{CCT} \gamma$ and $\mathrm{CCT} \eta$ [20]. In order to identify the CCT $\theta$ subunit in the 2-D gel, we have compared the putative $\mathrm{p} I$ values of the distinct TpCCT subunits so far known (see Table 1). Taking into account these results it is plausible to propose that spot P6 in the 2-D gel of Tetrahymena CCT (see Fig. 3) corresponds to TpCCT $\theta$ and CCTE is $\mathrm{P} 5$ as it has the most acidic pI. Moreover, Soares et al. [24] have observed that UM-1 antibody strongly recognize Hsp60, ТрССТ $\beta$, ТрССТ $\gamma$, ТрССТ $\eta$ and P5, whereas TpCCT $\alpha, \mathrm{P} 6$ and $\mathrm{P} 7$ spots were weakly recognized. In fact, UM-1 antibody was prepared against the chaperonin consensus motif which is highly conserved between all chaperonin sequences, that seems to be involved in ATP binding and hydrolysis [23]. When a comparison between the amino acid sequence used to produce the UM-1 antibody (QDDEVGDGTTSVV) and the corresponding sequence in TpCCT $\theta$ (QENECGDATNLVI) was done, we observe that these sequences only share six identical amino acids residues out of 13. This poor similarity can explain the weak signal for spot P6 (only visible after longer exposures) obtained when UM-1 antibody is used. It is worthwhile noting that CCT $\theta$ from mouse testis is also weakly recognized by UM-1 antibody [23], a fact that reinforces the idea that the UM1 antibody has different affinities for the distinct CCT subunits.

Total cytoplasmic RNAs from exponentially growing cells, cells recovering their cilia and cells treated with colchicine $(4 \mathrm{mg} / \mathrm{ml})$ at different times were ex-

Table 1

$\mathrm{p} I$ values predicted from the sequence analysis

\begin{tabular}{|c|c|c|c|c|c|}
\hline & $\mathrm{TpCCT} \alpha$ & ТрССТ & ТрСCT $\gamma$ & ТрССТ $\theta$ & $\mathrm{TpCCT \varepsilon}$ \\
\hline $\mathrm{p} I$ & 6.90 & 6.44 & 6.38 & 5.73 & 5.11 \\
\hline
\end{tabular}

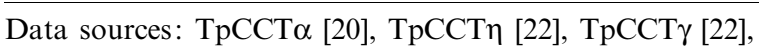
$\mathrm{TpCCT \varepsilon}$ (unpublished results). 
1

TрСCT $\theta$

$\operatorname{seC} T \theta$

$\operatorname{spCCT} \theta$

Сасст $\theta$

мmСст $\theta$

HsCCT $\theta$

трсCт $\theta$

SCCCT $\theta$

Sрсст $\theta$

Сасст $\theta$

MmCCT $\theta$

HsCCT $\theta$

TpCCT $\theta$

$\operatorname{SCCCT} \theta$

$\operatorname{spCCT} \theta$

Сасст $\theta$

Мm CCT $\theta$

HsCCT $\theta$

трсст $\theta$

ScCCT $\theta$

$\operatorname{spCCT} \theta$

CaCct $\theta$

$\mathrm{MmCCT} \theta$

HsCCT $\theta$

трсст $\theta$

SCCCT $\theta$

$\operatorname{spCCT} \theta$

СасСт $\theta$

МmCCT $\theta$

$H s \mathrm{CCT} \theta$

трсст $\theta$

SCCCT $\theta$

SpCCT $\theta$

СaCcT $\theta$

MmCCT $\theta$

HsCCT $\theta$

трсст $\theta$

$S C \operatorname{CT} \theta$

SpсCT $\theta$

Сасст $\theta$

$M m \operatorname{CCT} \theta$

HsCCT $\theta$

трсст $\theta$

SCCCT $\theta$

$\operatorname{spCCT} \theta$

Сасст $\theta$

$\mathrm{MmCCT} \theta$

HsCT $\theta$ MSLOSAYG INSLLKEGHR HFSGMEEALL KNINACKEIS NMTKTSLGPN GMKKMVINHI MSLR PQNPN. AG $F$ Q YN SY NADGQII S A IR LH Q CL M C RN IIV MALRVPK $S . P Q \quad F R \quad Y$ IMQ V D VI R C IR L EI R KN I V MSLK $P Q$ PN . SG $F$ Q YS $S$ NADG II R VE VR A SILL $M \quad S \quad R N$ IIV K $\begin{array}{lllllllllllllllll}\text { MALHVPK } & P & \text { FAQM } & D & \text { AK } & \text { L } & \text { VY } & R & Q & \text { LA } & Q T & R & A Y & N & R\end{array}$

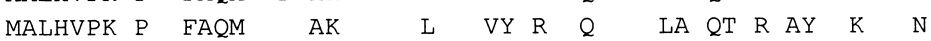

61

DKIFVTSDAA TIMOEMEVOH PAAKMTVMAS KMOENECGDA TNLVIATAGE ITSOAFSTIK G II $\mathrm{N}$ MLR LDIV V VL T EQ KIDM G N MI N QQT L N IR L I LV D T QQ L A A F VFS A $L K$ NM $R$ $G K I N \quad M L N$ L IV VV ILIQ $Q$ F M $N \quad$ I $\quad F \quad N V \quad K \quad L T$

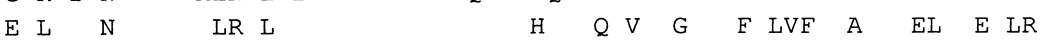

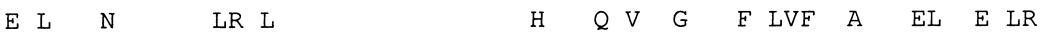

121 MGLHPSQIIT GYEQALKAAI ELLNTLSVFT VEDPTNLEQV SKALKASLSS KLIHHADFFA SAVE \& NM R FTL KE DEMV GE IT KNDKNEL L MI PVI KYGSE ILS $T$ LE AK $M$ SHTM $V$ EEICADK I TVESEKEL I IRTCI QYGNE LS L NV E Q FNL N FVM KT DE V EK SFE..TDL L V PVIAA QYGVE TI

I SV EV S I C K H I PE VCCS AKNLRDVDE SL RT IM QYGSET L I SV EV E I CRK H I PN VCCS AKNLRDIDE SL RT IM QYGNEV L

181

240 QIVAQACINS KPENDDT... ..FDLEEVRV AKILGASIED SYVQQGLIIT RNAEGSITRV EL SE VSHV L VAQQAGEI PY NVDSI $V$ M G LSN T IK MVFN EP HVKSL DL K ILTV L KDPSK... ... NVDNI V M S LYN Q VK MVFP EP TV $S$ KL VD VALV MKNGS....... . NVDNI V VM LSQ Q VK MVFP EP TVK.. KLI VSI F .DSGN... .. NVDNI C SG YS S LH MVFK KET DV $S$ KLI VSI $F$. DSGH... .. NVDNI C $\quad$ SG SS S LH MVFK KET DV $S$

241

300

SNPK. ..VAV YSCPLDTQQA ETKGTVLIQN AKELLNYTKS EESHAESIVK KIADSGINLI ED HK FT IANT LH Q M DFS G KQIDAMM E $\mathrm{M}$ VEC KEA ... F IS T LH $Q$ M DFS G NLI HI E Y A VRVV NADQIQSCR QPHRYFHHR NQRYSCSSTM PRKC ISPRA KNNSWT CAR STIQ LRWI

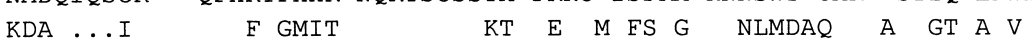
KDA ...I F GMIT KT E $M$ FS G NLMDAQ A $\quad$ T A VV

$301 \quad 360$ IAGGSISEIV LHYVEKYKMM IVKCQSKFEL KRICKALGAC PVARLDAPNP EEIGFCDSAS $V$ AGVG LA LNR GIL VL VP $R$ L RVC T LP G T $\quad$ L LVETVK

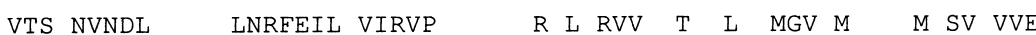
LP $S$ VG LA LN GIL VLRVP $D$ R QVC $T$ LP $G \quad T$ D M EI IIE VT KVAD A AN NI L RLN WD R L TV T ALPK TP VQ VT KVADMA AN NI L RLN WD R L TV T ALP TP VL $\mathrm{M}$ H

361 420

VEEIGSQKVT IIKKEN.QDC KINTIILRGS TINELDDIER AIDDGVNVYR CLLK. DGKF TM GDR VF Q QGEIS RTS A Q N AAVK G M PSG I TI GDR VFRQVE.DIT RTA $V$ A KTY IVK A V . NRL TK GDR FRQDESS.S RTA $V$ A $Q N \quad$ SIK G I .. NRL LS V DTQ V VF H K.E G AIS V D LM V V TEK V TR.. KRL LS V DTQ V VF H K.E G AIS V D LM V V TFK V TR.. KRL

421

480

AAGAGATEAV LSHKLQQQAK SLEDLSQYAF NRFALSFEII PRILSDNAGL NSNEIIPKLN LP IE ISRITKYGE RTPG L L I KQ VA VV T AET DV VL N Y IF SDMQ CIR ISVGE KTPGIY H I KQYGEA VV TI E DPTDV S Y LP V IE MKRITAY.Q TPG L L I KS KA $V \quad$ V AETS H D $S$ LS H

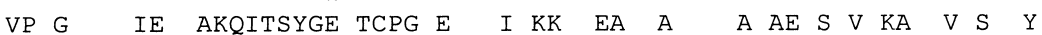
VP G IE AKQITSYGE TCPG E I KK EA A A AE S V KA $V$ S $\quad$ Y 


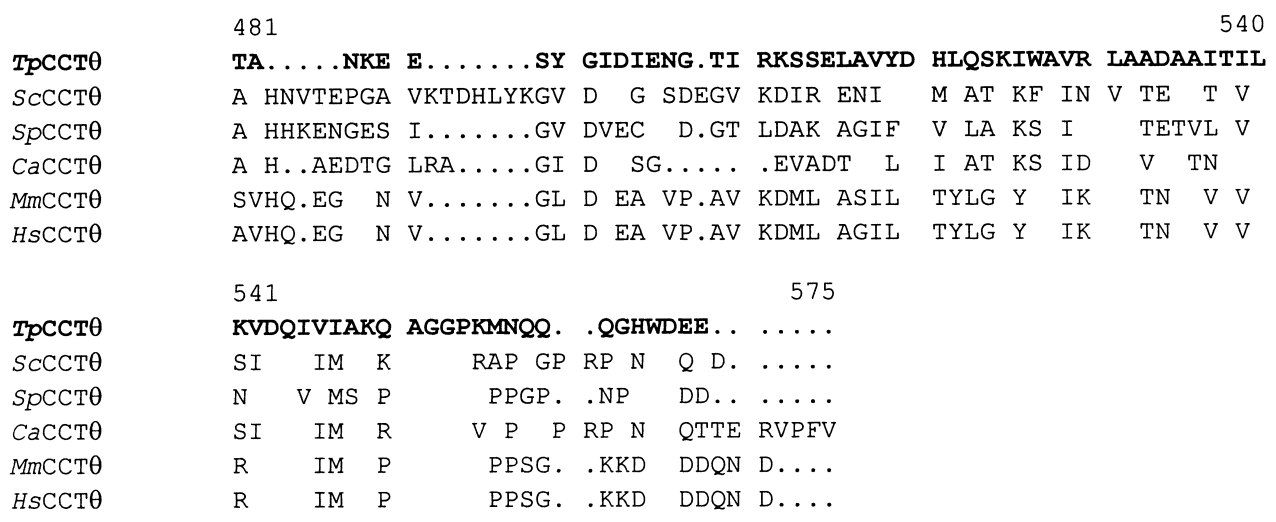

Fig. 2. Alignment and comparison of Tetrahymena TpCCT $\theta$ deduced amino acid sequence with CCT $\theta$ from other species. The deduced amino acid sequence of the $T$. pyriformis CCT $\theta$ polypeptide was aligned and compared with those of CCT $\theta$ from other organisms (accession numbers in the EMBL database are indicated): Saccharomyces cerevisiae (ScCCT $\theta$ - P47079), Schizosaccharomyces

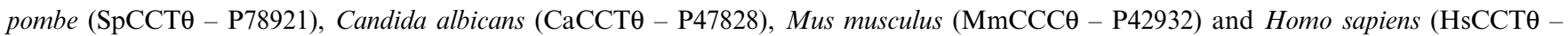
P50990). Gaps have been inserted when required to maximize the alignment and are represented by dots. Sequences conserved between almost all chaperonins, including the traditional chaperonins, are underlined. Sequence exclusively conserved between CCT $\theta$ subunit is double-underlined.

tracted from $T$. pyriformis cells as reported by Soares et al. [24]. RNAs were subjected to Northern blot analysis as described by Sambrook et al. [25] and the membranes were incubated with pCCT 051.5 $\mathrm{kb}$ fragment as probe in RNA hybridization buffer at $35^{\circ} \mathrm{C}$ [21]. The results show that TpCCT $\theta$ is expressed coordinately with the other TpCCT subunit genes (TpCCT $\alpha$, ТрCCT $\gamma$ and TpCCT $\eta)$ as well as

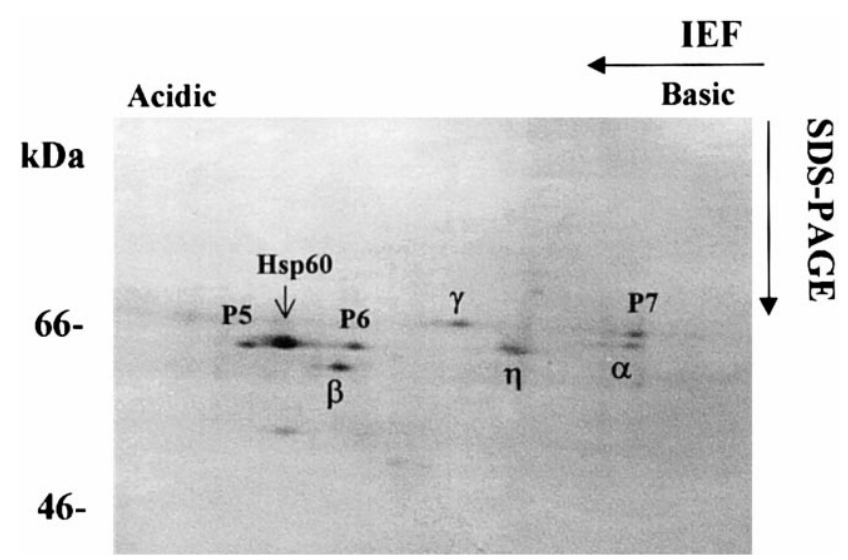

Fig. 3. Two-dimensional isoelectric focusing (IEF) and SDSPAGE analysis of Tetrahymena CCT from exponentially growing cells. Protein extracts containing CCT particles isolated from exponentially growing Tetrahymena cells by sucrose-gradient fractionation followed by ATP-affinity column chromatography were resolved by two-dimensional electrophoresis and detected by silver staining. The subunits TpCCT $\alpha, \mathrm{TpCCT \eta}$, ТрССТ $\gamma$ and ТрСCT $\beta$ where identified as described in [20]. with tubulin genes during cilia regeneration (results not shown) $[8,20,21]$. These results indicate that at least these four subunits are required for the function of the chaperonin during the biogenesis of the new cilia. We have also studied the expression of TpCCT $\theta$ in cells treated with a microtubule depolymerizing agent, colchicine $(4 \mathrm{mg} / \mathrm{ml})$. Results in Fig. 4a illustrate the hybridization pattern of the mRNA levels from exponentially growing cells and cells treated for different times with colchicine. As one can observe, TpCCT $\theta$ produces a unique transcript with about $2.1 \mathrm{~kb}$ during the whole period of colchi-

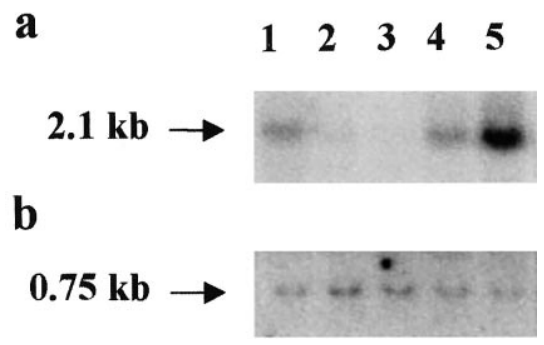

Fig. 4. TpCCT $\theta$ mRNA levels in Tetrahymena cells treated with colchicine. Total cytoplasmic RNA $(30 \mu \mathrm{g})$ from exponentially growing cells (lane 1) and from cells treated with colchicine (final concentration $4 \mathrm{mg} / \mathrm{ml}$ ) after $15 \mathrm{~min}$ (lane 2), $30 \mathrm{~min}$ (lane 3), $60 \mathrm{~min}$ (lane 4) and $90 \mathrm{~min}$ (lane 5), was analysed in $1.5 \%$ agarose formaldehyde gels, transferred onto nitrocellulose filters and hybridized (a) with pCCT $\theta 51.5 \mathrm{~kb}$ fragment as probe and (b) with the 239 bp PstI-SphI DNA fragment from pTU11 [26]. 
cine treatment, as well as in control cells. The amount of the steady-state population of the CCT $\theta$ mRNA decreases rapidly until $30 \mathrm{~min}$ of colchicine treatment as compared to control cells. However, the amount of the steady-state population of this transcript significantly increases after $60 \mathrm{~min}$, reaching levels higher than those found in exponentially growing cells for up to $90 \mathrm{~min}$ of drug treatment. Densitometric quantification of the relevant bands indicates a 1.3- and 3.5-fold increase of the levels at 60 and 90 min of treated cells, respectively, compared to exponentially growing cells. As a loading and hybridization control we used the 239 bp PstI-SphI DNA fragment isolated from the ubiquitin fusion gene as probe in the same RNA blot [26]. The results obtained (Fig. 4b) show that the respective mRNA levels do not change during colchicine treatment.

The fact that colchicine binds specifically to the tubulin heterodimer, modifying the exchange rate of tubulin at microtubule ends, suggests that the TpCCT $\theta$ gene is able to change its pattern of expression in response to an alteration of microtubule polymerization. Experiments are in progress with the aim of elucidating the potential meaning of the colchicine effect.

This work was supported by grants from Calouste Gulbenkian Foundation to C.R.-P. and from PRAXIS/PCNA/BIO/67/96.

\section{References}

[1] R.J. Ellis, S.M. Van der Vies, Molecular chaperons, Annu. Rev. Biochem. 60 (1990) 321-347.

[2] F.U. Hartl, Molecular chaperones in cellular protein folding, Nature 381 (1996) 571-579.

[3] R.J. Ellis, Chaperonins: introductory perspective, in: R.J. Ellis (Ed.), Chaperonins, Academic Press, London, 1996, pp. $1-25$.

[4] J.D. Trent, E. Nimmesgern, J.S. Wall, F.-U. Hartl, A.L. Horwich, A molecular chaperone from a thermophilic archaebacterium is related to the eukaryotic protein $t$-complex polypeptide-1, Nature 354 (1991) 490-493.

[5] B.M. Phipps, A. Hoffmann, K.O. Stetter, W. Baumeister, A novel ATPase complex selectively accumulated upon heat shock is a major cellular component of thermophilic archaebacteria, EMBO J. 10 (1991) 1711-1722.

[6] V.A. Lewis, G.M. Hynes, D. Zheng, H. Saibil, K. Willison, T-complex polypeptide-1 is a subunit of a heteromeric particle in the eukaryotic cytosol, Nature 358 (1992) 249-252.
[7] V. Stoldt, F. Rademacher, V. Kehren, J.F. Ernest, D.A. Pearce, F. Sherman, The Ccct eukaryotic chaperonin subunits of Saccharomyces cerevisiae and other yeasts: a mini review, Yeast 12 (1996) 523-529.

[8] H. Soares, D. Penque, C. Mouta, C. Rodrigues-Pousada, A Tetrahymena orthologue of the mouse chaperonin subunit $\mathrm{CCT} \gamma$ and its coexpression with tubulin during cilia recovery, J. Biol. Chem. 269 (1994) 29299-29307.

[9] H. Kubota, K. Willison, A. Ashworth, M. Nozaki, H. Miyamoto, A. Yamamoto, A. Matsushiro, A. Morita, Structure and expression of the gene encoding $t$-complex polypeptide 1 (Tcp-1), Gene 120 (1992) 207-215.

[10] J. Frydmann, E. Nimmesgern, H. Erdjument-Bromage, J.S. Wall, P. Tempst, F.-U. Hartl, Function in protein folding of TRIC, a cytosolic ring complex containing TCP-1 and structurally related subunits, EMBO J. 11 (1992) 4767-4778.

[11] Y. Gao, I. Vainberg, R.L. Chow, N.J. Cowan, Two cofactors and cytoplasmic chaperonin are required for the folding of $\alpha$ - and $\beta$-tubulin, Mol. Cell. Biol. 13 (1993) 24782485.

[12] Y. Gao, J.O. Thomas, R.L. Chow, G.-H. Lee, N.J. Cowan, A cytoplasmic chaperonin that catalyses $\beta$-actin folding, Cell 69 (1992) 1043-1050.

[13] K.R. Willison, G. Hynes, P. Davies, A. Goldsborough, V.A. Lewis, Expression of three t-complex genes, Tcp-1, D17Leh117c3 and D17Leh66, in purified murine spermatogenic cell populations, Gen. Res. Camb. 56 (1990) 193-201.

[14] H. Kubota, G. Hynes, K. Willison, The chaperonin containing $t$-complex polypeptide 1 (TCP-1). Multisubunit machinery assisting in protein folding and assembly in the eukaryotic cytosol, Eur. J. Biochem. 230 (TCP-1) (1995) 3-16.

[15] X. Chen, D.S. Sullivan, T.C. Huffaker, Two yeast genes with similarity to TCP-1 are required for microtubule and actin function in vivo, Proc. Natl. Acad. Sci. USA 91 (1994) 91119115.

[16] D.B.-N. Vinh, D.G. Drubin, A yeast TCP-1 like protein is required for actin function in vivo, Proc. Natl. Acad. Sci. USA 91 (1994) 9116-9120.

[17] R.H. Wade, A.A. Hyman, Microtubule structure and dynamics, Curr. Opin. Cell Biol. 9 (1997) 12-17.

[18] D.A. Skoufias, L. Wilson, Mechanism of inhibition of microtubule polymerization by colchicine: inhibitory potencies of unliganded colchicine and tubulin-colchicine complexes, Biochemistry 31 (1992) 738-746.

[19] D. Panda, J.E. Daijo, M.A. Jordan, L. Wilson, Kinetic stabilization of microtubule dynamics at steady state in vitro by substoichiometric concentrations of tubulin-colchicine complex, Biochemistry 34 (1995) 9921-9929.

[20] H. Soares, L. Cyrne, C. Casalou, B. Ehmann, C. RodriguesPousada, The third member of the Tetrahymena CCT subunit gene family, $T p C C T \alpha$, encodes a component of the hetero-oligomeric chaperonin complex, Biochem. J. 326 (1997) 21-29.

[21] L. Cyrne, P. Guerreiro, A.C. Cardoso, C. Rodrigues-Pousada, H. Soares, The Tetrahymena chaperonin subunit CCTn gene is coexpressed with CCT $\gamma$ gene during cilia biogenesis 
and cell sexual reproduction, FEBS Lett. 383 (1996) $277-$ 283.

[22] H. Kubota, G. Hynes, K. Willison, The eighth Cct gene, Cctq, encoding the theta subunit of the cytosolic chaperonin containing TCP-1, Gene 154 (1995) 231-236.

[23] G. Hynes, H. Kubota, K.R. Willison, Antibody characterisation of two distinct conformations of the chaperonin-containing TCP-1 from mouse testis, FEBS Lett. 358 (1995) 129-132.

[24] H. Soares, L. Galego, R. Cóias, C. Rodrigues-Pousada, The mechanisms of tubulin messenger regulation during Tetrahymena pyriformis reciliation, J. Biol. Chem. 268 (1993) 1662316630.

[25] J. Sambrook, E.F. Fritsch, T. Maniatis, Molecular Cloning: A Laboratory Manual, Cold Spring Harbor Laboratory, Cold Spring Harbor, NY, 1992.

[26] A.M. Neves, P. Guerreiro, L. Miquerol, C. Rodrigues-Pousada, Molecular cloning and expression of a Tetrahymena pyriformis ubiquitin fusion gene coding for a 53-amino-acid extension protein, Mol. Gen. Genet. 230 (1991) 186-192. 